\title{
Clinical neurophysiology and imaging of nerve injuries: preoperative diagnostic work-up and postoperative monitoring
}

\author{
Andrea Gagliardoㄹ, Francesca Toia², Francesco Maggì², Alessio Vincenzo Mariolo², \\ Michele Cillino' ${ }^{2}$ Francesco Moschella ${ }^{2}$ \\ 1"Clinical Course" Neurophysiology Unit, NHS Accredited, 90146 Palermo, Italy. \\ ${ }^{2}$ Plastic and Reconstructive Surgery, Department of Surgical, Oncological and Oral Sciences, University of Palermo, 90127 Palermo, Italy.
}

Address for correspondence: Dr. Francesca Toia, Plastic and Reconstructive Surgery, Department of Surgical, Oncological and Oral Sciences, University of Palermo, 90127 Palermo, Italy. E-mail: francescatoia@gmail.com

\begin{abstract}
Peripheral nerve injuries are a heterogeneous group of lesions that may occurs secondary to various causes. Several different classifications have been used to describe the pathophysiological mechanisms leading to the clinical deficit, from simple and reversible compression-induced demyelination, to complete transection of nerve axons. Neurophysiological data localize, quantify, and qualify (demyelination vs. axonal loss) the clinical and subclinical deficits. High-resolution ultrasound can demonstrate the morphological extent of nerve damage, fascicular echotexture (epineurium vs. perineurium, focal alteration of the cross-section of the nerve, any neuromas, etc.), and the surrounding tissues. High field magnetic resonance imaging provides high contrast neurography by fat suppression sequences and shows structural connectivity through the use of diffusion-weighted sequences. The aim of this review is to provide clinical guidelines for the diagnosis of nerve injuries, and the rationale for instrumental evaluation in the preoperative and postoperative periods. While history and clinical approach guide neurophysiological examination, nerve conduction and electromyography studies provide functional information on conduction slowing and denervation to assist in monitoring the onset of re-innervation. High-resolution nerve imaging complements neurophysiological data and allows direct visualization of the nerve injury while providing insight into its cause and facilitating surgical treatment planning. Indications and limits of each instrumental examination are discussed.
\end{abstract}

Key words:

Electromyography, imaging, injury, magnetic resonance imaging, nerve conduction studies, neurodiagnostic, peripheral nerve, ultrasound

\section{INTRODUCTION}

Every year more than $5 \%$ of patients admitted to a level one trauma center have a concurrent traumatic peripheral nerve injury. ${ }^{[1]}$ These patients are often young

\begin{tabular}{|l|l|}
\hline \multicolumn{2}{|c|}{ Access this article online } \\
\hline Quick Response Code: & Website: \\
\hline & www.parjournal.net \\
\cline { 2 - 2 } & \\
\hline
\end{tabular}

adults at the peak of their employment productivity, and therefore, functional decline associated with nerve lesions is particularly significant. ${ }^{[2]}$ Thus, there is a great interest in optimizing both the diagnostic accuracy and early treatment of peripheral nerve injuries.

The purpose of this review is to discuss peripheral nerve injuries and their diagnostic management and outcomes evaluation with regard to clinical findings and neurodiagnostic studies and imaging.

The goal is to provide a practical guide for general management that is, applicable to all types of nerve injuries. The main classifications and basic principles of a correct clinical approach will be summarized. Next, 
the indications and correct timing for each instrumental examination will be reviewed, with a specific focus on innovative methods and future prospects.

\section{CLASSIFICATION OF PERIPHERAL NERVE INJURIES}

The most commonly used classification for peripheral nerve injuries is that by Seddon, ${ }^{[3]}$ and Sunderland..$^{[4]}$ The Seddon classification places injuries into three basic types: neurapraxia, axonotmesis, and neurotmesis.

Neurapraxia (praxis: to do, to perform): the nerve axons are intact but cannot transmit impulses. This occurs secondary to ischemic damage with temporary myelin sheath damage. Without myelin, there is an alteration of "saltatory conduction" across the nodes of Ranvier with subsequent slowed or blocked nerve conduction. Neuropraxia is the mildest form of nerve injury; "Saturday night" radial palsy and entrapment neuropathies like carpal tunnel syndrome is good example for this condition. ${ }^{[5,6]}$ Nerve recovery occurs after remyelination and sensory-motor functions can usually completely restored within days to weeks. ${ }^{[7]}$

Axonotmesis (tmesis: to cut): the axons are damaged or destroyed, but most of the connective scaffold (endoneurium, perineurium, and epineurium) remains intact. Axonotmesis is commonly seen in crush and stretch injuries. ${ }^{[8]}$ After injury, anterograde Wallerian degeneration of the distal axonal fibers is completed within a few days.

Neurotmesis: the nerve trunk is disrupted and loses anatomical continuity. Neurotmesis represents the most severe form of injury with disruption of the axons, myelin sheath, and connective tissues. It may occur following sharp injuries, massive trauma, or severe traction that partially or completely interrupts nerve continuity. ${ }^{[9]}$ In order to enhance the chances for reinnervation after neurotmesis, surgical nerve repair is mandatory. ${ }^{[10]}$ Without surgery, uncontrolled axonal re-growth will generate a neuroma.

The Sunderland classification includes five stages and identifies three types of neurotmesis: (1) stage I corresponds to neuropraxia; (2) stage II corresponds to axonotmesis; and (3) stages III, IV, and V correspond to neurotmesis [Table 1], with impairment of the endoneurium, perineurium, and epineurium.

The distinction between the different types of injury is not always precise. Clinical evaluation benefits from instrumental approaches to discriminate severity at an earlier stage, thus allowing for appropriate and timely treatment.

\section{CLINICAL APPROACH}

Patient age, mechanism of injury and associated vascular and soft tissue injuries strongly influence the extent of recovery of the injured nerve. These elements are of great importance and are the primary details collected in the clinical history. A detailed examination includes evaluation of pain and muscular strength and sensory testing in the territory of the injured nerve. The homologous contralateral and other ipsilateral preserved nerves are used for comparison, particularly in polytrauma patients. ${ }^{[11]}$ Appropriate motor and sensory evaluation is mandatory to identify injuries to sensitive, motor, and mixed nerves; early and late signs of autonomic disorders should also be evaluated, including vasomotor disorders and trophic alteration of the skin, nails, and subcutaneous tissue. ${ }^{[11,12]}$ Both negative (e.g. hypoesthesia, muscle weakness, and atrophy) and positive symptoms (e.g. dysesthesia, pain, fasciculations) due to loss of nerve function or inappropriate spontaneous activity, respectively, should be noted.

The simplest standardized clinical evaluation of a cutaneous somatic sensitivity is the test of the pain pathway (the patient's ability to perceive the touch of a sharp object). ${ }^{[13 \mid}$ Clinicians and surgeons generally refer to cutaneous nociception because of less lower overlap of innervating territories when compared to tactile sensation.

Hypoesthesia generally involves all superficial and deep somatosensory systems (tactile, thermal, pain, and proprioception); anatomical charts and diagrams help to

Table 1: Classification of peripheral nerve injuries according to Seddon and Sunderland

\begin{tabular}{|c|c|c|c|c|c|}
\hline $\begin{array}{l}\text { Type of injury } \\
\text { Seddon classification }\end{array}$ & $\begin{array}{l}\text { Type of Injury } \\
\text { Sunderland } \\
\text { classification }\end{array}$ & $\begin{array}{l}\text { Major structure } \\
\text { involved }\end{array}$ & Prognosis & Neurodiagnostic findings & $\begin{array}{l}\text { Requirement } \\
\text { for surgical } \\
\text { intervention }\end{array}$ \\
\hline Neuropraxia & I & Myelin & Good & $\begin{array}{l}\text { Slower conduction velocity } \\
\text { or conduction block; EMG } \\
\text { with no fibrillation, reduced } \\
\text { recruitment and fast firing }\end{array}$ & None \\
\hline Axonotmesis & II & $\begin{array}{l}\text { Myelin, } \\
\text { Axons }\end{array}$ & $\begin{array}{l}\text { Fair } \\
\text { (depending on how many } \\
\text { fibers are involved) }\end{array}$ & $\begin{array}{l}\text { Reduced CMAP and } \\
\text { SNAP amplitudes; EMG } \\
\text { with fibrillation, reduced } \\
\text { recruitment and fast firing }\end{array}$ & $\begin{array}{l}\text { Depends on } \\
\text { extension of } \\
\text { the lesion }\end{array}$ \\
\hline Neurotmesis & $\begin{array}{l}\text { III, } \\
\text { IV, } \\
\text { V }\end{array}$ & $\begin{array}{l}\text { Myelin, } \\
\text { Axons, } \\
\text { Endoneurium } \\
\text { Perineurium } \\
\text { Epineurium }\end{array}$ & $\begin{array}{l}\text { Poor } \\
\text { (depending on how many } \\
\text { fibers are involved) }\end{array}$ & $\begin{array}{l}\text { Reduced or absent CMAP } \\
\text { and SNAP; EMG with } \\
\text { fibrillation and motor units } \\
\text { loss }\end{array}$ & $\begin{array}{l}\text { Often requires } \\
\text { surgical repair }\end{array}$ \\
\hline
\end{tabular}

EMG: Electromyography, CMAP: Compound muscle action potential, SNAP: Sensory nerve action potential 
identify areas that correspond to specific nerves or to dermatomes (useful for root or spinal level injuries).

Sensory disorders may also include positive (irritative) symptoms which that should be explored: (1) paresthesia (spontaneous feeling of needles, tingling, numbness, and electric shock); (2) dysesthesia and hyperalgesia (inaccurate interpretation of a sensory stimulus which is perceived as different and with an affective unpleasant sensation); and (3) neuropathic pain (spontaneous pain consequent to a lesion in the afferent somatosensory fibers coming from the cutaneous territory of a nerve).

Motor signs and symptoms as a consequence of a reduced number of functional motor units include: (1) hyposthenia: reduced muscle strength as described by the use of the British Medical Research Council scale that recognizes five grades of muscle strength: 0 , neither contraction nor movement are visible; 1 , minimal contraction visible or flickering (residual functioning motor units) without movement; 2, active movement possible only without gravity (i.e. in a horizontal plane); 3 , active movement obtained against gravity; 4, active movement against mild resistance (4-), moderate resistance (4) or strong resistance (4+); and 5, normal strength; ${ }^{[14]}$ (2) muscular hypotrophy or atrophy: reduced volume of the muscle belly for both axonal damage and disuse; it will reach its maximum state in 3-4 months with a potential strength reduction of $80 \%$. If denervation persists, a proliferation of fibroblasts characterizes the histological picture, as new collagen is deposited in both the endo- and perimysium, and atrophied muscle fibers are replaced by thickened connective tissue; (3) absence or reduction of osteotendinous (phasic) reflexes and of muscular tone (tonic reflex) due to involvement of both afferent sensory fibers from muscular spindles and efferent motor neuron axons of the somatic arc reflex; (4) hyposthenia, hypotrophy, and hypotonia configure the picture of partial or total flaccid paralysis of the group of muscles innervated by the affected nervous structures (roots, plexus, nerves); (5) positive symptoms (fasciculations and cramps) are rare in peripheral nerve injuries, but are often seen in radiculopathies; and (6) deformities: in chronic and severe cases, muscle paresis reduced joint movement in conjunction with healthy muscles may lead to deformities (cavus foot, claw-hand) and ankylosis.

No clinical evaluation can distinguish neurapraxia from axonotmesis, and no clinical or neurophysiological examination can distinguish axonotmesis from neurotmesis. To obtain the correct diagnosis and a plan appropriate to treatment, both neurophysiological and imaging studies and clinical re-evaluation over time are often required.

\section{CLINICAL NEUROPHYSIOLOGICAL STUDIES}

The neurophysiological or neurodiagnostic study represents an extension of the clinical examination; accordingly, neurodiagnostic tests should always be combined with a directed neurologic examination, in order to identify the clinical abnormalities and establish a differential diagnosis. For this reason, the evaluation is commonly referred as the clinical neurophysiological examination.

Clinical neurophysiological examination is currently the gold standard for diagnosis and determination of prognosis in peripheral nerve injuries, ${ }^{[15,16]}$ in order to localize and quantify clinical and subclinical preoperative damage and postoperative recovery. As such, it yields key information on the type of involved fibers (sensory vs. motor), on the underlying pathophysiology (demyelination vs. axonal loss), on axonal loss quantification, and consequently on prognosis.

The core neurodiagnostic studies are nerve conduction studies and electromyography (EMG). These tools test the integrity and physiological function of peripheral sensory and motor fibers and the muscles.

In order to reveal axonal loss (presence of denervation potentials), the optimal timing of a neurodiagnostic study is 2-3 weeks after injury. ${ }^{[17,18]}$ Neurodiagnostic studies should be repeated 3 months or more following trauma or surgical repair to assess the ratio of denervation to reinnervation. ${ }^{[19]}$

\section{Nerve conduction studies}

Nerve conduction studies are the first line studies in instrumental evaluation of nerve injuries. They are the most basic and easily performed types of neurodiagnostic studies, and also used for screening prior to any additional testing. ${ }^{[20]}$

Nerves and muscles are excitable structures and their potentials can be induced and recorded by external electrodes. When the nerve is stimulated, a compound muscle action potential (CMAP) can be recorded from the muscle, and a nerve action potential (NAP) can be recorded from the nerve. Amplitude and latency of the evoked response and conduction velocity are analyzed. ${ }^{[21]}$

The amplitude of the evoked response estimates the quantity of depolarized motor or sensory fibers, while conduction velocity measures the speed of the fastest (and large caliber) motor or sensory myelinated axons.

Sensory NAPs (SNAPs) are also helpful in differentiating between preganglionic (radiculopathies) and postganglionic lesions; postganglionic lesions produce abnormal SNAP due to Wallerian degeneration of the axons distal to the peripheral injury, whereas in preganglionic lesions axon degeneration occurs in the dorsal root and in the ascending central pathway, leaving peripheral fibers intact and SNAP unmodified, despite anesthesia in the examined cutaneous territory. ${ }^{[21]}$

Caution should be paid to interpretation of pure or prevalent motor diseases. Although changes in the CMAP are frequently used to preliminarily diagnose peripheral nerve injuries, they are not specific and may reveal, spinal disease of the anterior horn cells (myelopathy, amyotrophic lateral sclerosis, etc.), myopathy (muscular 
dystrophy, myositis, etc.), a myelin-related acquired or congenital disorders (chronic inflammatory demyelinating polyneuropathy, Charcot-Marie-tooth disease) ${ }^{[22]}$ or presynaptic neuromuscular junction disorders (Eaton-Lambert syndrome, botulism)

In neurapraxia, nerve conduction is either slowed or blocked secondary to demyelination. With stimulation proximal to the lesion, the conduction velocity will be reduced (conduction slowing), or the evoked potential amplitude will drop with respect to the normal potential obtained by distal stimulation (conduction block). When nerve remyelination completes, these abnormalities progressively disappear, with eventual complete recovery.

In the case of axonotmesis and neurotmesis, after distal axonal degeneration (which completes in 3-5 days for motor fibers and in 6-10 days for sensory fibers), CMAP and SNAP are reduced in amplitude when stimulating distally to the injury; the ratio between CMAP/SNAP amplitudes on the injured side to the CMAP/SNAP of the normal side is a good estimate of the degree of axonal loss. The higher the axonal loss, the lower the odds of recovery.

For technical reasons, exploration of the proximal peripheral nervous system is more complex; late responses such as $\mathrm{F}$ waves and the $\mathrm{H}$ reflex can be obtained for further information and somatosensory or motor evoked potentials can be explored. ${ }^{[23,24]}$

\section{Electromyography}

This examination requires the active participation of the patient. Needle EMG provides information on the function of the muscles function and their minimal functional units. It explores both the quantity and quality of motor unit action potentials (MUAP), their spatial-temporal recruitment in order to generate adequate movements, the presence of denervation, and the onset of re-innervation. ${ }^{[18]}$ In partial or gradual denervation, reinnervation occurs early through collateral sprouting by adjacent surviving axons. In nerve transection, the only mechanism available for re-innervation is axonal regrowth from the proximal stump of the injury site. This regrowth is slow (1 $\mathrm{mm} /$ day) and may take months to years to reach the target muscles, depends on the distance to be covered.

The first step in EMG of nerve injuries is the evaluation of pathological potentials at rest. Fibrillation potentials and positive sharp waves are the most common potentials and appear 10-21 days after injury, while complex repetitive discharges indicate chronic and ongoing denervation. Although all these potentials are a sign of muscle fiber denervation, they can also be found in myopathies and myositis, which also induce hyposthenia. Fasciculation potentials occur from the spontaneous activation of motor units (all muscle fibers innervated by one neuron), which can be visualized directly as minor muscle twitches. Cramps are a painful involuntary contraction of the muscle which tend to occur when a muscle is in the shortened position and contracting, and can be recorded as a firing of motor unit potentials at high frequency.
Many other spontaneous potential can be recorded from muscles, but their discussion is beyond the intent of this review.

The following step in the neurophysiological examination is the analysis of MUAP and their activation and recruitment patterns during voluntary contraction.

In acute axonal loss and pure demyelinating nerve injuries with conduction block, not all motor units can be recruited; the remaining MUAPs have normal morphology but fire with high frequency in order to obtain sufficient contraction, and the recruitment pattern results in incomplete interference. Note that denervation potentials will appear only in case of axonal damage.

In chronic axonal loss and denervation, early collateral sprouting from re-innervation of orphan muscle fibers by surviving axons is recorded on EMG as small satellite potentials of the MUAP's. Later, as the number of muscle fibers per motor unit increases with re-innervation, MUAP's become higher in amplitude, prolonged in duration, and polyphasic; these are the typical neurogenic MUAP's representing the pattern of denervation and reinnervation.

Incomplete nerve transection and in late stages of partial axonal loss, if regrowing axons from the site of injury eventually reach the target, very small low-voltage nascent MUAP potentials will be recorded. As reinnervation occurs, denervation potentials will gradually disappear.

\section{NERVE IMAGING TECHNIQUES}

Neurophysiological investigation offers information on the pathophysiology of the nerve deficit, the grade of severity, and prognosis. Although it is a fundamental tool in clinical evaluation, it does not provide precise information on the morphology, etiology or the extent of focal peripheral nerve injuries versus the focal involvement of only few fascicles.

In severe cases with unexcitable nerves and in postoperative patients who do not shows signs of improvement, EMG and conduction velocities cannot provide conclusive information on the presence of neurotmesis, nerve transection, the distance between nerve stumps, and the presence of multiple sites of injury. ${ }^{[25]}$ Imaging assessment, in particular high-resolution ultrasound (HRU) and magnetic resonance imaging (MRI), may overcome these problems by providing information on nerve morphology and its surrounding tissues; these are becoming popular instruments for planning nerve reconstruction and the surgical approach.

\section{High-resolution ultrasound}

Although MRI is still more commonly used, based on our experience and on a review of the recent literature, the authors believe that HRU currently represents the most easily available and practical imaging technique for investigation of peripheral nerve pathology [Figures 1 and 2]. These machines are widely available and, when associated with high frequency transducers (7-18 MHz), reach up 


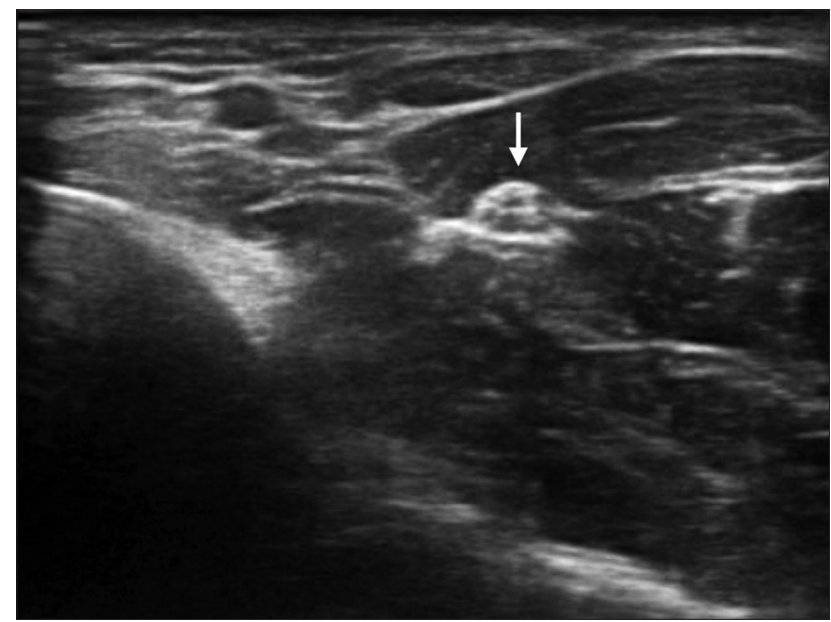

Figure 1: Axial scan of median nerve (arrow) at mid forearm; note the fascicular texture of the nerve and the homogeneous echogenicity of the surrounding muscles

to $400 \mu \mathrm{m}$ in axial resolution, which is higher than that achieved by a common MRI. ${ }^{[26]}$ There is increasing evidence in the literature on the helpfulness of HRU, in particular in cases with equivocal clinical and neurophysiological data; ${ }^{[27]}$ HRU may be diagnostic in a significant percentage of such patients. ${ }^{[28]}$ Its advantages include a bedside, painless study of the nerve along the entire limb, with color-Doppler analysis integration and dynamic scans. In addition, it can be utilized in the presence of metal implants and orthopedic screws, and therefore is preferable to a high-cost, single segment MRI study.

Sonographic criteria for nerve identification are based on fascicular echotexture detection. ${ }^{[26]}$ The cross-sectional area (CSA) of the nerve is one of the most studied parameters and is examined in each nerve along the length of the limb in an axial scan. CSA measurements are performed at the inner border of the thin hyperechoic rim of the nerve, ${ }^{[29]}$ across the site of entrapment or trauma to calculate the distal-proximal CSA ratio. The nerve CSA is significantly related to the neurodiagnostic data and, when performed side by side with a comprehensive neurodiagnostic exam, it increases its diagnostic sensitivity. ${ }^{[30,31]}$

Echogenicity of the nerve should be reported; an increased CSA of the entire nerve or of a few fascicles, proximal to the site of entrapment or trauma, can be associated with fibrosis of the fascicles or epineurium. A few nerve pathologies, such as Schwannomas, will initially spare the nerve's conduction and sensory-motor functions, manifesting only with inconstant signs of irritation. Fiber sparing and dislocation can be recognized by an experienced HRU examiner.

Finally, nerve continuity can be assessed based on the analysis of the epi-perineurium and on the presence of a partial neuroma or transection. ${ }^{[32]}$

Imaging will also uncover any predisposing anatomic abnormalities (i.e. bifid median nerve or persistent median artery) or other concurrent diseases in the surrounding tissues which may require a different therapeutic approach. Examples include space-occupying lesions, tumors, tenosynovitis, osteophytes, neurovascular

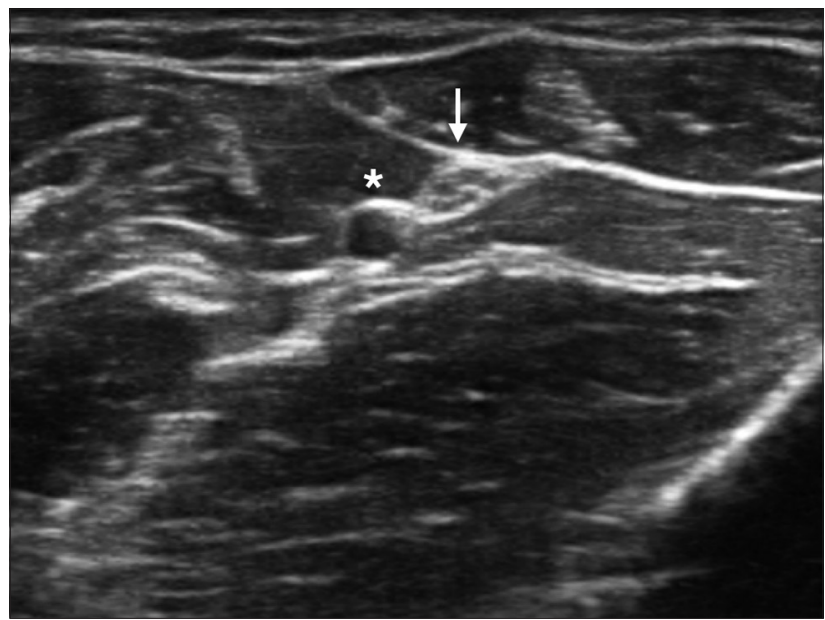

Figure 2: Axial scan of ulnar nerve (arrow) and ulnar artery (*) at forearm; in live scans pulsating arteries are a good landmark to be recognized

conflicts, abnormal muscles or muscle insertions, synovial cysts, nerve subluxation, postfracture fibrosis, and bone formation.

Neurophysiological and clinical parameters are good predictors of postsurgical recovery, but HRU has also demonstrated its usefulness when correlated with clinical neurophysiology in several nerve pathologies: (1) in patients with a history of trauma, it can reveal neuromas and neurotmesis; (2) in cases of postsurgical neuropathy of an iatrogenic origin, uncommon sites of injury can be localized; (3) in severe diseases with unevocable nerve potentials on neurophysiological examination, the site of injury can be easily showed by ultrasound; (4) in patients with diffuse preexisting (and confounding) neurophysiological alterations and clinical signs of a new neuropathy, the nerve lesions can be delineated; (5) in entrapment neuropathies, for screening purposes (e.g. concomitant tenosynovitis is seen in $21.7 \%$ of carpal tunnel syndromes, and dynamic ulnar nerve subluxation is seen at the elbow in $28.5 \%$ of cubital grooves); (6) in all brachial plexus pathologies, to identify multiple sites of injury are common; (7) for early selection of surgical candidates; ${ }^{[33,34]}$ and (8) for detection of postsurgical improvement or complications. ${ }^{[35]}$

HRU does have some limitations, high frequency probes provide optimal spatial resolution for superficial nerve imaging while the deeper nerve course may remain unexplored. ${ }^{[36]}$ The sciatic nerve trunk cannot be investigated over the horizontal gluteal fold, and the tibial and common peroneal nerves cannot be easily examined in the mid leg behind the calf. Both the deep nerve segments and nerve roots emerging from the spine should be explored by MRI. Expert HRU investigation can be used to visualize the cervical roots of the brachial plexus (the anterior branches of the spinal nerves as they emerge from the intervertebral foramen) as well as the trunks in the interscalene area and the cords in the supraclavicular and infraclavicular and axillary regions. A similar guide is helpful in interventional procedures to reach target nerves, such as in regional anesthesia or during steroid infiltrations, thus minimizing the risk of complications. 
Ultrasound is already in use for a number of indications in the evaluation of nerves and is likely to find increasing indications in the future. ${ }^{[37-39]}$ However, further clinical and biomedical research is required to further validate its application in preoperative and postoperative monitoring.

\section{Magnetic resonance imaging}

MRI is appreciated mainly for its wide overview of the limb with the option of selective volume reconstruction. Direct nerve visualization by MRI has also been optimized; ${ }^{[0,41]}$ "MR neurography" combines fat suppression T2-weighted sequences and diffusion weighting in high magnetic field gradients (1.5T or higher). The nerve's signal increases significantly following traumatic nerve injury, resulting in high contrast of the bright nerve (hyperintense) against the surrounding muscle or fat. The increased nerve signal due to axonal degeneration can be observed both at the site of the injury and distally, and is the single most searched MRI sign for localization of nerve injuries. However, it is not a specific sign, reflecting only endoneurial or perineural edema and slower axoplasmic transport secondary to axonal degeneration.

Diffusion-weighting imaging has the potential to detect structural anisotropy by determination of the main orientation of the axons within the nerves; this method is called diffusion tensor imaging (DTI). From DTI data, a three-dimensional reconstruction of major fascicles can be rendered and is referred to as "tractography". ${ }^{42]}$ Tractography provides structural information on the nerves, but has low spatial resolution and a low signal-to-noise ratio, adding no additional information to neurophysiological data.

Many techniques including MRI myelography, MR neurography, and DTI can be combined for additional data, for example in root avulsions in patients with brachial plexus injuries, ${ }^{[43]}$ but in order for the higher sequences to be carried out, greater acquisition times are required.

To overcome current limitations of MRI and enable investigation of nerves along a limb with faster image acquisition, widespread upgrade to 3T scanners combined with parallel imaging will be required.

Future application of new technologies for nerve imaging such as very high field magnetic fields (9.4T) MRI, ${ }^{[4]}$ or very high frequency ultrasound probes $(55 \mathrm{MHz})^{[45]}$ will also increase spatial resolution up to a theoretical histological precision of $30 \mu \mathrm{m}$.

At this time, HRU provides the highest spatial resolution of direct nerve imaging along the limbs, while MRI provides a high contrast delineation of preselected single segments of the body. Both can assist in the resolution of pitfalls in injury localization, which may arise if only certain proximal nerve fascicles are injured, and others are spared, simulating a distal neuropathy.

\section{CONCLUSION}

Evaluation of peripheral nerve injuries remains a challenge for both clinicians and surgeons. A comprehensive clinical and physical examination approach permits formulation of a differential diagnosis to guide the neurophysiological exam and estimate prognosis. Nerve imaging evaluation completes the work-up by visualizing fascicles and continuity of the nerve and its surrounding tissue.

Clinical and instrumental data should be integrated to plan adequate treatment and promote functional recovery. High-resolution nerve imaging, when correlated with neurophysiological data, provides the missing link to clinicians and surgeons, closing the gap between diagnostic and therapeutic approaches. To optimize prognosis, this comprehensive evaluation is mandatory not only during the preoperative stage, but also during follow-up in order to recognize late or non-recovery, thus preventing permanent neurological disability.

\section{REFERENCES}

I. Taylor CA, Braza D, Rice JB, Dillingham T. The incidence of peripheral nerve injury in extremity trauma. Am J Phys Med Rehabil 2008;87:38I-5.

2. di Summa PG, Kalbermatten DF, Pralong E, Raffoul W, Kingham PJ, Terenghi G. Long-term in vivo regeneration of peripheral nerves through bioengineered nerve grafts. Neuroscience 201 I;|81:278-9|.

3. Seddon HJ. A classification of nerve injuries. Br Med J 1942;2:237-9.

4. Sunderland $\mathrm{S}$. The anatomy and physiology of nerve injury. Muscle Nerve 1990; | 3:77|-84.

5. Gupta R, Rummler L, Steward O. Understanding the biology of compressive neuropathies. Clin Orthop Relat Res 2005;436:25I-60.

6. Gupta R, Rowshan K, Chao T, Mozaffar T, Steward O. Chronic nerve compression induces local demyelination and remyelination in a rat model of carpal tunnel syndrome. Exp Neurol 2004; 187:500-8.

7. Bernsen HJ, Koetsveld A, Frenken CW, van Norel GJ. Neuropraxia of the cervical spinal cord following cervical spinal cord trauma: a report of five patients. Acta Neurol Belg 2000;100:91-5.

8. Lee SK,Wolfe SW. Peripheral nerve injury and repair. JAm Acad Orthop Surg 2000;8:243-52.

9. Menorca RM, Fussell TS, Elfar JC. Nerve physiology: mechanisms of injury and recovery. Hand Clin 20I3;29:3 I7-30.

10. Koeppen $\mathrm{AH}$. Wallerian degeneration: history and clinical significance. J Neurol Sci 2004;220: I I5-7.

II. Kleggetveit IP, Jørum E. Large and small fiber dysfunction in peripheral nerve injuries with or without spontaneous pain. J Pain 2010;1 I:1305-10.

12. Intiso D, Grimaldi G, Russo M, Maruzzi G, Basciani M, Fiore P, Zarrelli M, Di Rienzo F. Functional outcome and health status of injured patients with peripheral nerve lesions. Injury 2010;41:540-3.

13. Pinelli P, Poloni M. Neurology. Principles of Diagnosis and Therapy. 3rd ed. Rozzano: Casa Editrice Ambrosiana; 2003. p. 75-87.

14. Medical Research Council of the UK. Aids to the investigation of peripheral nerve injuries. Memorandum No. 45. London: Pendragon House; 1976. p. 6-7.

15. Aminoff MJ. Electrophysiologic testing for the diagnosis of peripheral nerve injuries. Anesthesiology 2004;100:1298-303.

16. Robinson LR. Traumatic injury to peripheral nerves. Muscle Nerve 2000;23:863-73.

17. Bergquist ER, Hammert WC. Timing and appropriate use of electrodiagnostic studies. Hand Clin 2013;29:363-70.

18. Kane NM, Oware A. Nerve conduction and electromyography studies. J Neurol 2012;259:1502-8.

19. Campbell WW. Evaluation and management of peripheral nerve injury. Clin Neurophysiol 2008; 1 19:1951-65.

20. Gutmann L, Pawar GV. An approach to electrodiagnosis of peripheral neuropathies. Semin Neurol 2005;25:160-7.

21. Fisher MA. Electrophysiology of radiculopathies. Clin Neurophysiol 2002; I I3:3 |7-35

22. Perry JD. Electrodiagnosis in musculo-skeletal disease. Best Pract Res Clin Rheumatol 2005; 19:453-66.

23. Restuccia $D$, Valeriani M, Di Lazzaro V, Tonali P, Mauguière F. Somatosensory evoked potentials after multisegmental upper limb stimulation in diagnosis of cervical spondylotic myelopathy. J Neurol Neurosurg Psychiatry 1994;57:30 I-8. 
24. Le Pera D, Valeriani M, Tonali P, Restuccia D. Selective abnormality of the NI3 spinal SEP to dermatomal stimulation in patients with cervical monoradiculopathy. Neurophysiol Clin 1998;28:221-9.

25. Gagliardo A, Avarino C, Giaimi G, Di Matteo D, Midiri M, Gagliardo C. Emerging Role of Ultrasound Imaging Associated to Clinical Neurophysiology as an Advanced Diagnostics of Peripheral Nerves Pathologies. A Sicilian Experience. Neuroradiology, 37th European Society of Neuroradiology Annual Meeting; 2013 September 28, October I; Frankfurt, Germany. Berlin: Springer; 2013. p. SII4.

26. Koenig RW, Schmidt TE, Heinen CP, Wirtz CR, Kretschmer T, Antoniadis G, Pedro MT. Intraoperative high-resolution ultrasound: a new technique in the management of peripheral nerve disorders. J Neurosurg 20 I I; I |4:5 I 4-2I.

27. Beekman R, Visser LH. Sonography in the diagnosis of carpal tunnel syndrome: a critical review of the literature. Muscle Nerve 2003;27:26-33.

28. Padua L, Aprile I, Pazzaglia C, Frasca G, Caliandro P, Tonali P, Martinoli C. Contribution of ultrasound in a neurophysiological lab in diagnosing nerve impairment: a one-year systematic assessment. Clin Neurophysiol 2007; I 18:1410-6.

29. Cartwright MS, Passmore LV, Yoon JS, Brown ME, Caress JB, Walker FO. Cross-sectional area reference values for nerve ultrasonography. Muscle Nerve 2008;37:566-7I.

30. Klauser AS, Halpern EJ, De Zordo T, Feuchtner GM, Arora R, Gruber J, Martinoli C, Löscher WN. Carpal tunnel syndrome assessment with US: value of additional cross-sectional area measurements of the median nerve in patients versus healthy volunteers. Radiology 2009;250:I7I-7.

31. Bayrak AO, Bayrak IK, Turker H, Elmali M, Nural MS. Ultrasonography in patients with ulnar neuropathy at the elbow: comparison of cross-sectional area and swelling ratio with electrophysiological severity. Muscle Nerve 2010;41:66I-6.

32. Huang Y, Zhu J, Liu F. Ultrasound in diagnosis of retroperitoneal femoral nerve injury: a case report. J Plast Reconstr Aesthet Surg 2013;66:e50-2.

33. Gagliardo A, Avarino C, Giaimi G, Di Matteo D, Midiri M, Gagliardo C. Ultrasound combined with clinical neurophysiology in peripheral nerve pathologies: when it is worth? Preliminary data in 50 outpatients. Clin Neurophysiol 2013;124:el89.

34. Gagliardo A, Coraci D, Romano M, Fernandez Marquez EM, Tsukamoto $H$, de Franco P, Padua L. Clinical, neurophysiological and ultrasound assessment in post-surgical follow up of nerve injuries. A case report. Clin Neurophysiol 2013;124:e222.

35. Zhu J, Liu F, Li D, Shao J, Hu B. Preliminary study of the types of traumatic peripheral nerve injuries by ultrasound. Eur Radiol 201 I;21:1097-I0I.

36. Torres C, Mailley K, Del Carpio O'Donovan R. MRI of the brachial plexus: modified imaging technique leading to a better characterization of its anatomy and pathology. Neuroradiol J 2013;26:699-7I9.

37. Wang Y, Zhao C, Passe SM, Filius A, Thoreson AR, An KN, Amadio PC. Transverse ultrasound assessment of median nerve deformation and displacement in the human carpal tunnel during wrist movements. Ultrasound Med Biol 2014;40:53-6I.

38. Klauser AS, Tagliafico A, Allen GM, Boutry N, Campbell R, Court-Payen M, Grainger A, Guerini H, McNally E, O'Connor PJ, Ostlere S, Petroons P, Reijnierse M, Sconfienza LM, Silvestri E, Wilson DJ, Martinoli C. Clinical indications for musculoskeletal ultrasound: a Delphi-based consensus paper of the European Society of Musculoskeletal Radiology. Eur Radiol 2012;22: I 140-8.

39. Kerasnoudis A. Which ultrasound method has the upper hand in the follow-up of the patients with recurrent carpal tunnel syndrome? Ann Rheum Dis 20I3;72:ell.

40. Filler AG, Howe FA, Hayes CE, Kliot M, Winn HR, Bell BA, Griffiths JR, Tsuruda JS. Magnetic resonance neurography. Lancet 1993;341:659-6I.

4I. Howe FA, Filler AG, Bell BA, Griffiths JR. Magnetic resonance neurography. Magn Reson Med 1992;28:328-38.

42. Hiltunen J, Suortti T, Arvela S, Seppä M, Joensuu R, Hari R. Diffusion tensor imaging and tractography of distal peripheral nerves at $3 \mathrm{~T}$. Clin Neurophysiol 2005; I 16:2315-23.

43. Gasparotti R, Lodoli G, Meoded A, Carletti F, Garozzo D, Ferraresi S. Feasibility of diffusion tensor tractography of brachial plexus injuries at I.5 T. Invest Radiol 2013;48:104-12.

44. Bilgen M, Heddings A, Al-Hafez B, Hasan W, Mclff T, Toby B, Nudo R, Brooks WM. Microneurography of human median nerve. J Magn Reson Imaging 2005;21:826-30.

45. Kuffler DP. Ultrasound imaging of regenerating rat sciatic nerves in situ. J Neurosci Methods 2010;188:276-9.

How to cite this article: Gagliardo A, Toia F, Maggì F, Mariolo AV, Cillino M, Moschella F. Clinical neurophysiology and imaging of nerve injuries: preoperative diagnostic work-up and postoperative monitoring. Plast Aesthet Res 2015;2:149-55.

Source of Support: Nil, Conflict of Interest: None declared.

Received: 10-04-2015; Accepted: 11-06-2015 